\title{
Atuação das academias de ginástica durante a pandemia da Covid-19
}

\author{
Performance of gymnastics academies during the Covid-19 pandemic \\ J. A. Araújo Júnior ${ }^{1}$; G. Mendonça ${ }^{2 *}$; J. J. O. Toscano ${ }^{1 *}$ \\ ${ }^{1}$ Instituto de Educação Física e Esporte - IEFE, Núcleo de Estudos Aplicados em Atividade Fúsica e Saúde - \\ NEAAFIS, Universidade Federal de Alagoas, Campus A.C. Simões, 57072-900, Maceió-AL, Brasil \\ ${ }^{2}$ Curso de Educação Física do Centro Universitário CESMAC e do Instituto de Educação Física e Esporte -IEFE \\ Universidade Federal de Alagoas, Campus A.C. Simões, 57072-900, Maceió-AL, Brasil
}

*jean.toscano@hotmail.com.br

(Recebido em 03 de setembro de 2020; aceito em 19 de outubro de 2020)

\begin{abstract}
O objetivo do estudo foi descrever o conteúdo de práticas de exercícios físicos veiculado virtualmente por academias de ginástica durante a pandemia da COVID-19. Trata-se de estudo descritivo, observacional e com característica retrospectiva. A amostra foi composta por oito academias de ginástica distribuídas nas regiões administrativas do município de Maceió - AL. Foi elaborado um formulário para registrar as variáveis de interesse: plataformas virtuais utilizadas, tipo das postagens, conteúdo e o número de visualizações dessas postagens nos meios virtuais utilizados no período de abril, maio e junho de 2020. Foram realizadas análises descritivas das variáveis contínuas por meio da média e valores mínimos e máximos. Para as variáveis categóricas, foi utilizada a distribuição de frequências em números absolutos e relativos. Nos resultados, foi identificado um total de 91.836 seguidores dos perfis de redes sociais das oito academias observadas. Foram identificadas um total de 449 tipos distintos de postagens, sendo a maioria realizada na rede social Instagram $(85,0 \%)$; o conteúdo mais prevalente foram os posts informativos $(64,5 \%)$. Em relação ao conteúdo especifico de vídeo aulas ou lives de treinamento, as atividades com perfil de demanda cardiorrespiratória (aeróbicas) foram as que apresentaram maior prevalência de oferta $(47,3 \%)$, seguido das atividades com capacidades mistas (aeróbias e resistidas) com 32,3\%. As academias de ginástica em seus ajustes póspandemia, além dos cuidados com a biossegurança, podem se aproximar mais dos seus clientes utilizando, de modo mais efetivo, tecnologias virtuais.
\end{abstract}

Palavras-chave: academias de ginástica, pandemias, redes sociais online

The aim of the study was to describe the content of physical exercise practices transmitted virtually by fitness centers during the COVID-19 pandemic. Descriptive, observational study, with retrospective characteristic. The sample consisted of eight fitness centers distributed in the administrative regions of the city of Maceió AL. A form was created to record the variables of interest: virtual platforms used, type of posts, content and the number of views of these posts in the virtual media used in the period of April, May and June 2020. Descriptive analyzes of the continuous variables were carried out by average and minimum and maximum values. For categorical variables, the distribution of frequencies in absolute and relative numbers was used. In the results, a total of 91,836 followers of the social network profiles of the eight observed gyms were identified. A total of 449 different types of posts were identified, the majority of which were carried out on the social network Instagram (85.0\%); the most prevalent content was informative posts (64.5\%). Regarding the specific content of video classes or training lives, activities with a profile of cardiorespiratory demand (aerobics) were the ones with the highest prevalence of supply (47.3\%), followed by activities with mixed capacities (aerobic and resisted) with 32.3\%. Gymnasiums in their post-pandemic adjustments, in addition to caring for biosafety, can get closer to their customers using more effectively, virtual technologies.

Keywords: fitness centers, pandemics, online social networking

\section{INTRODUÇÃO}

Com a declaração da Organização Mundial de Saúde (OMS), em março de 2020, de uma pandemia de gripe causada pelo novo coronavírus (Sars-Cov-2), vários países, incluindo o Brasil, adotaram medidas de isolamento e distanciamento social como um padrão de enfrentamento à COVID-19 [1-2]. Em Alagoas, o governo estadual, publicou o primeiro decreto de medidas de 
restrição no dia 20 de março de 2020 (Decreto $\mathrm{N}^{\circ} 69.541$ de 20/03/2020), suspendendo o funcionamento de vários setores da sociedade, dentre eles, as academias de ginástica [3].

Esforços em diversas frentes foram realizados no sentido de manter as pessoas fisicamente ativas. Na cidade de Wuhan, China, epicentro inicial da doença, o governo local recomendou a população manter-se fisicamente ativa em casa [4]. O American College Sports of Medicine (ACSM), logo após a OMS decretar pandemia, reuniu em sua página na internet um vasto material sobre dicas para manter-se fisicamente ativo [5]. No Brasil, tendo como referência o documento do ACSM, o Centro de Estudos do Laboratório de Aptidão Física de São Caetano do Sul (CELAFISCS) publicou um material informativo intitulado: Quarentena Sim! Sedentarismo Não! [6].

Nesse contexto de fechamento de academias de ginástica, centros esportivos, parques públicos e orla marítima durante a COVID-19, a relação do movimento humano como fator de proteção à saúde recebeu grande destaque, não apenas da literatura cientifica, mas do jornalismo de modo geral [7]. Um estudo da consultoria especializada App Annie mostrou que o número de downloads dos aplicativos nas categorias saúde e fitness saltou, após o início do isolamento, de 1,2 milhão para 4,4 milhões por semana no Brasil [8]. Além disso, startups ganharam mais espaço no país, nesse período da pandemia, com a proposta do usuário pagar uma mensalidade para ter acesso a diversas atividades, a serem acessadas quando quisessem através da televisão, smartphone ou computador, proposta semelhante ao streaming de vídeo em canais de assinatura como a Netflix e Amazon Prime.

As academias de ginástica constituem os principais centros de prestação de serviço para a prática de exercícios físicos sob supervisão especializada, principalmente entre a população adulta [9]. De acordo com o relatório da International Health, Racquet \& Sportsclub Association (IHRSA) Global Report 2019, associação que reúne informações sobre o desenvolvimento do mercado de fitness em todo o mundo, o Brasil permanece como o segundo maior mercado do mundo em número de academias, atrás apenas dos Estados Unidos [10].

Diante desse inesperado contexto histórico da pandemia, como as academias de ginástica se mobilizaram para atender seus clientes? Esse trabalho teve por objetivo descrever o conteúdo veiculado virtualmente por academias de ginástica durante a pandemia da COVID-19.

\section{MATERIAL E MÉTODOS}

Esse trabalho foi do tipo descritivo, observacional, com característica retrospectiva. A população alvo do estudo foi composta por academias de ginástica localizadas no município de Maceió - AL. Para delimitação da amostra, a capital alagoana foi dividida em oito regiões administrativas. Em cada uma dessas regiões foi selecionada, por conveniência, uma academia, permanecendo ao final oito academias.

Foi elaborado um formulário para registrar as variáveis de interesse desse estudo: tipos de plataformas virtuais utilizadas (Instagram/Facebook, Zoom, Microsoft Tems, Meet ou aplicativos próprios, o conteúdo postado (posts informativos, vídeo-aulas, lives, palestras, vídeos curtos), a frequência das postagens e o número de visualizações. Nesse formulário foram registradas, quantitativamente, as informações postadas semanalmente pelos meios virtuais utilizados pelas academias de ginástica no período de abril, maio e junho de 2020.

Inicialmente em cada região administrativa, foi feita uma busca de academias através do Google Maps (inserindo o termo "academia de ginástica"), em seguida foi verificado se a academia selecionada tinha perfil oficial em seu nome nas redes sociais, por fim, se esses meios virtuais estavam ativos no período da pandemia. A pós a identificação, foi feito contato com o responsável legal da academia e realizado o convite para participação no estudo. A confirmação de aceite pelo responsável se deu por meio do preenchimento e assinatura do Termo de Consentimento Livre e Esclarecido (TCLE). O projeto para o presente estudo foi submetido e aprovado pelo Comitê de ética da Universidade Federal de Alagoas (UFAL) sob o parecer n. 4.186.358.

Os dados foram digitados em planilha do Excel e revisada a consistência e amplitude das variáveis. Todos os erros foram identificados e corrigidos com base nos valores registrados nos questionários. Foram realizadas análises descritivas das variáveis contínuas por meio da média e 
valores mínimos e máximos. Para as variáveis categóricas, foi utilizada a distribuição de frequências em números absolutos $\left(\mathrm{n}^{\circ}\right)$ e relativos $(\%)$. As análises estatísticas foram efetuadas no Stata 14.0.

\section{RESULTADOS}

Os resultados são referentes as oito academias participantes desse estudo, distribuídas em seis das oito regiões administrativas do município de Maceió (em duas regiões não foi possível incluir academias, ficando a região $3 \mathrm{com}$ mais de uma academia representada). Foi identificado um total de 91.836 seguidores dos perfis de redes sociais, sendo uma média de $11.479,5$ por academia (mínimo 2.971 e máximo 27.900 seguidores) (Figura 1).

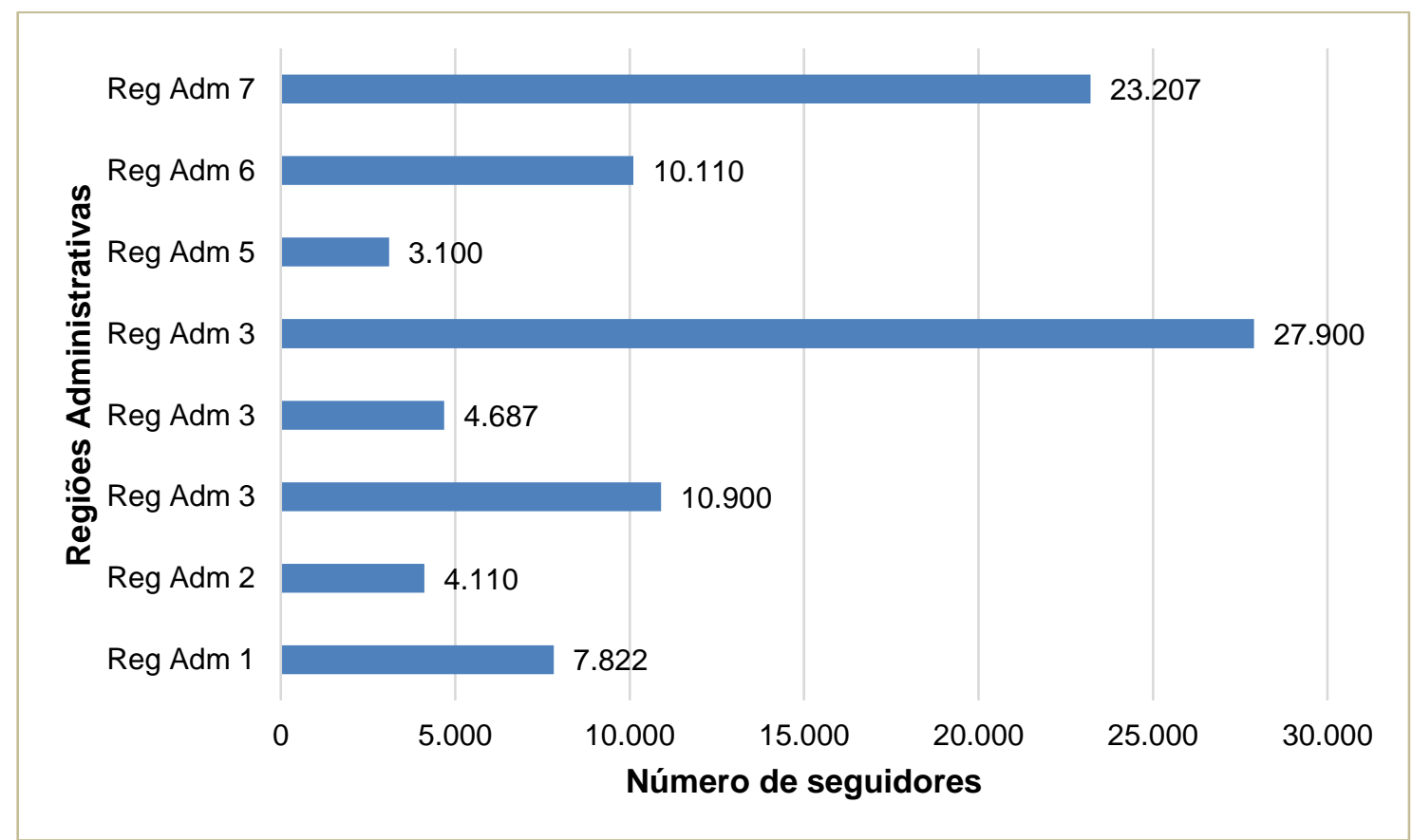

Figura 1: Total de seguidores nas redes sociais por academias participantes em seus respectivos distritos sanitários durante a pandemia de COVID-19-Maceió-AL.

No período de três meses de observações (abril, maio e junho) das atividades realizadas nos perfis das redes sociais das academias, foram identificadas um total de 449 tipos distintos de postagens, sendo a maioria realizada na rede social Instagram/Facebook (85\%; Tabela 1). O conteúdo das postagens foram agrupados da seguinte forma: posts motivacionais (fotos de exercícios, informações sobre a programação da academia, dicas sobre alimentação saudável, informativo de suplementos e frases motivacionais), esses tiveram a maior prevalência (64,5\%); em seguida vieram os vídeos curtos (informação referente à programação semanal da academia, demonstração de exercícios, dicas sobre alimentação) representando (24,0\%); as vídeo-aulas/lives (reproduzindo as modalidades de exercícios ofertadas na academia) foram 10,5\% das postagens (Tabela 1). Ao final do período de análise foram registradas um total de 35.612 visualizações (Tabela 1), resultando em uma média de 11.871 visualizações mensais entre as academias. 
Tabela 1: Prevalência das plataformas usadas pelas academias e conteúdo de postagens virtuais durante a pandemia de COVID-19-Maceió-AL.

\begin{tabular}{|c|c|c|c|c|c|c|c|c|}
\hline \multirow{2}{*}{ Variáveis em estudo } & \multicolumn{2}{|c|}{$1^{\circ}$ Mês } & \multicolumn{2}{|c|}{$2^{\circ}$ Mês } & \multicolumn{2}{|c|}{$3^{\circ}$ Mês } & \multicolumn{2}{|c|}{ Total } \\
\hline & $\mathrm{n}$ & $\%$ & $\mathrm{n}$ & $\%$ & $\mathrm{n}$ & $\%$ & $\mathrm{n}$ & $\%$ \\
\hline \multicolumn{9}{|l|}{ Plataforma Virtual } \\
\hline Instagram / Facebook & 31 & 32,3 & 26 & 27,1 & 39 & 40,6 & 96 & 85,0 \\
\hline Zoom & 1 & 7,1 & 4 & 28,6 & 9 & 64,3 & 14 & 12,3 \\
\hline App próprio & 1 & 33,3 & 1 & 33,3 & 1 & 33,3 & 3 & 2,7 \\
\hline \multicolumn{9}{|l|}{ Tipos de Postagens } \\
\hline $\begin{array}{l}\text { Vídeo-aulas e Lives de treinamento }(\geq 30 \\
\text { min.) }\end{array}$ & 6 & 12,8 & 14 & 29,8 & 27 & 57,4 & 47 & 10,5 \\
\hline Vídeos curtos (<30 min) & 28 & 25,7 & 35 & 32,1 & 46 & 42,2 & 109 & 24,3 \\
\hline Palestras & 1 & 33,3 & 2 & 66,7 & 0 & 0,0 & 3 & 0,7 \\
\hline Posts motivacionais / dicas / informativos* & 90 & 31,0 & 115 & 39,7 & 85 & 29,3 & 290 & 64,5 \\
\hline Número de visualizações & 10.927 & 30,7 & 10.334 & 29,0 & 14.351 & 40,3 & 35.612 & 100,0 \\
\hline
\end{tabular}

* Informações diversas sobre saúde (beber água frequentemente, para uma melhor noite de sono), dietas, suplementos, filmes e horários das atividades.

No que se refere as atividades propostas por meio das vídeo-aulas e lives para treinamento, identificou-se que 47,3\% das atividades ofertadas tinham um perfil de demanda cardiorrespiratória (aeróbicas) e 32,3\% das práticas trabalhavam com atividades mistas (aeróbio e resistido). Quanto a frequência das práticas, identificou-se que a maioria das academias $(81,7 \%)$ ofereciam suas práticas por meio de vídeo-aulas três ou mais vezes por semana (Tabela 2).

Tabela 2: Distribuição das academias em relação a frequência das postagens por tipo de atividade durante a pandemia de COVID-19-Maceió-AL.

\begin{tabular}{|c|c|c|c|c|c|c|c|c|}
\hline \multirow{2}{*}{$\begin{array}{l}\text { Variáveis videoaulas de } \\
\text { treinamento (>30 min.) }\end{array}$} & \multicolumn{2}{|c|}{$1^{\circ}$ Mês } & \multicolumn{2}{|c|}{$2^{\circ}$ Mês } & \multicolumn{2}{|c|}{$3^{\circ}$ Mês } & \multicolumn{2}{|c|}{ Total } \\
\hline & $\mathrm{n}$ & $\%$ & $\mathrm{n}$ & $\%$ & $\mathrm{n}$ & $\%$ & $\mathrm{n}$ & $\%$ \\
\hline \multicolumn{9}{|l|}{ Tipo } \\
\hline Aeróbio & 41 & 33,3 & 56 & 45,5 & 26 & 21,1 & 123 & 47,3 \\
\hline Resistido & 10 & 18,9 & 34 & 64,2 & 9 & 17,0 & 53 & 20,4 \\
\hline Misto (aeróbio + resistido) & 39 & 46,4 & 32 & 40,0 & 13 & 13,6 & 84 & 32,3 \\
\hline
\end{tabular}

Ao identificar o tipo de atividade quanto a sua especificidade energética, a Figura 2 descreve o tipo quanto as modalidades ofertadas pelas academias por meio de vídeo-aulas aos seus respectivos clientes. Dentre as modalidades mais ofertadas durante as vídeo-aulas/lives, destacou-se as postagens atividades relacionadas a coreografias com música: 37,9\%, seguida de treinamento funcional $(36,0 \%)$, seguida por (Figura 2). 


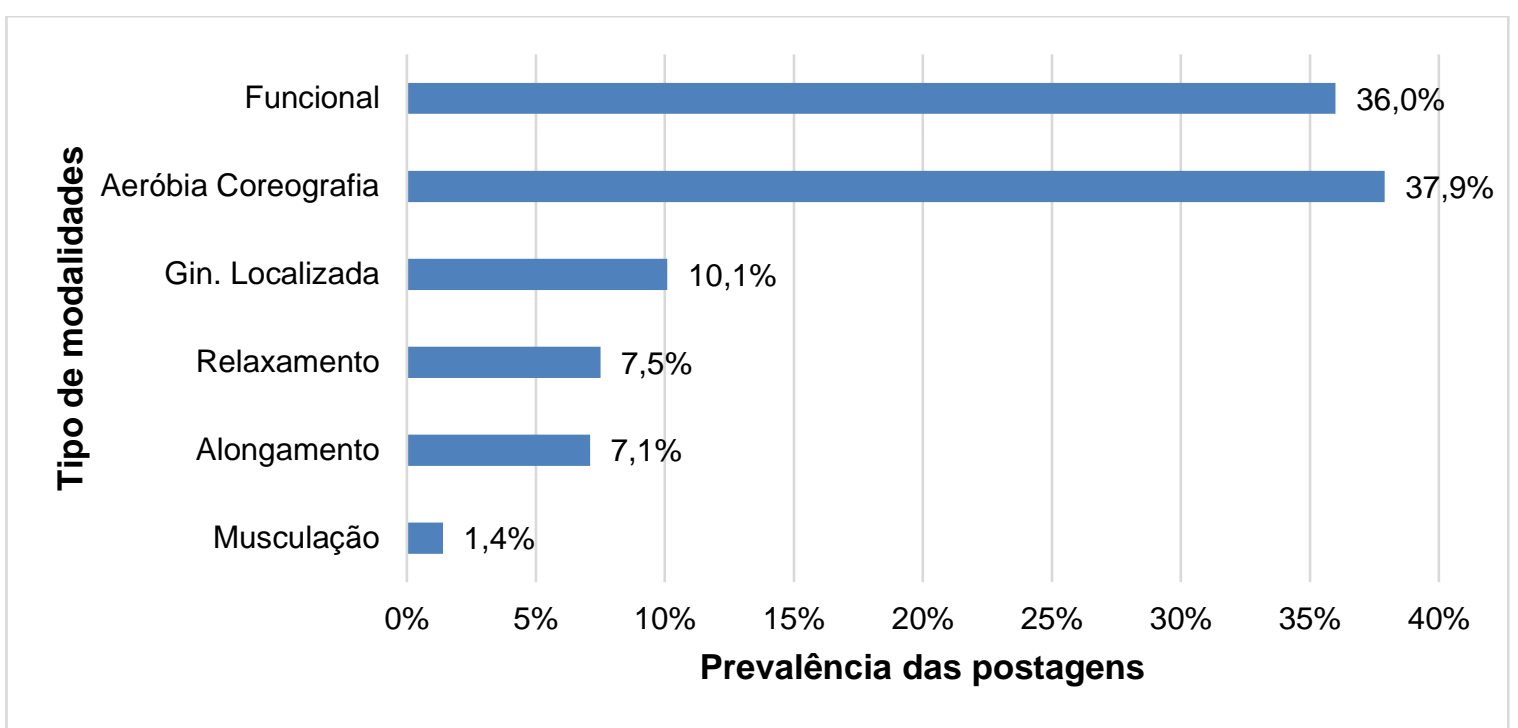

Figura 2: Prevalência de vídeo-aulas/lives de acordo com as modalidades de prática ofertadas durante a pandemia de COVID-19-Maceió-AL.

\section{DISCUSSÃO}

O presente trabalho teve como principal objetivo verificar o conteúdo veiculado virtualmente pelas academias de ginástica de Maceió-AL durante a pandemia de COVID-19. De forma geral, as academias de ginástica dispunham de uma conexão virtual com seus clientes já estabelecida através de seus endereços oficiais, somando um total de mais de noventa mil perfis, com destaque para o uso da mídia social Instagram/Facebook.

A literatura recente tem referenciado a utilização de novas tecnologias de informação e comunicação para transmitir mensagens sobre a importância da atividade física para a saúde e qualidade de vida das pessoas [11]. Os autores têm destacado que será um grande desafio o direcionamento não apenas das mensagens sobre a prática da atividade física, mas também a possibilidade de sistematização e viabilidade prática em possibilitar oferta de exercício físico de forma estruturada para atingir o público alvo que está vinculado a uma determinada tecnologia.

Verificamos no presente estudo que as mensagens veiculadas através de posts e vídeos curtos foram os meios mais utilizados pelas academias durante o período observado, e que em ambas, o conteúdo principal foi composto por orientações e dicas motivacionais para manter os clientes fisicamente ativos e informados sobre aspectos relacionados ao estilo de vida saudável. Nesse contexto de pandemia, acredita-se que informações pertinentes sobre a importância do exercício físico como fator proteção e na terapêutica de doenças crônicas, inclusive no sistema imune, sejam estratégias viáveis para aumentar o conhecimento sobre a relação do exercício físico o processo saúde-doença [12].

Entender como empregar mensagens sobre atividade física e saúde de maneira eficaz, utilizando tecnologias de informação e comunicação, bem como qual o conteúdo a ser elaborado, pode ser um importante passo para otimizar conteúdos direcionados aos subgrupos que utilizam os serviços das academias de ginástica. É importante destacar que através das mídias sociais, as informações têm seu alcance ampliado, podendo chegar a dezenas de milhares de pessoas [11], como as mais de 35 mil visualizações identificadas nas postagens pelas academias de ginástica observadas nos três meses do presente estudo.

Nesse contexto de isolamento social e respectivo fechamento das academias de ginástica, centros esportivos, parques públicos e orlas durante a pandemia, foi possível verificar na internet o crescimento na busca por "treinamento em casa" por parte da população em todo o mundo [13]. Nesse sentido, merece destaque as intervenções realizadas pelas academias de ginástica por meio de vídeo-aulas gravadas e/ou lives para atenderem a demanda de um "treinamento em casa". No presente estudo, observou-se que a maioria das academias de ginástica explorou atividades que desenvolviam o componente aeróbio, seguido daqueles que tinham um componente misto do tipo 
aeróbio com resistido. Nas atividades aeróbias as modalidades mais exploradas foram as coreografadas com música, nas mistas o treinamento funcional e nas resistidas a ginástica localizada.

Qual seria a razão para a grande preocupação da população, em especial aqueles com vínculo com academias de ginástica, querer manter-se fisicamente ativo. Em levantamento online realizado em maio de 2020 pelo startup Opinion Box abrangendo todas as regiões do Brasil, a motivação principal dos brasileiros que se exercitavam durante a pandemia era o cuidado com a "saúde" (70\%), seguido do cuidado com a "cabeça/mente" (47\%), a preocupação com a "aparência" apareceu em quinto lugar (30\%) [14].

Considerando o interesse com a saúde por parte da população e a crescente demanda para a prática de exercícios físicos, logo após a OMS decretar a situação de pandemia, uma das mensagens mais difundidas pelas mídias de modo geral, foi que as pessoas se mantivessem fisicamente ativas, sendo inclusive apresentado orientações de como realizar exercícios no ambiente doméstico [5].

No entanto, para um treinamento individualizado, como é a proposta da academia, as pessoas que procuram seus serviços provavelmente não querem apenas acumular semanalmente 150 minutos de atividade física aeróbia, de intensidade moderada, na maioria dos dias da semana, incluindo aí pelo menos dois dias de atividades de força muscular [15]. Talvez a demanda das pessoas que procuram as academias de ginástica seja por volumes diferenciados, objetivando atender interesses da aptidão física ou algum indicador de saúde que requeira atenção específica [9].

A preocupação do mercado fitness em fazer uma aproximação do off-line (estrutura física) com o online (tecnologias virtuais) já vem sendo identificada há alguns anos [16]. Resultados da pesquisa anual do ACSM com profissionais da área do fitness, sobre tendências de consumo, apontam a wearable technology (tecnologia vestível, em uma tradução literal) como primeira colocada para 2020 num ranking com vinte tendências, isso desde que entrou na lista em 2016 (excetuando 2018, terceiro lugar) e, recentemente foram incluídas outras terminologias como: online training e mobile exercise apps, mostrando uma tendência de crescimento nesse mercado [16].

Dispositivos eletrônicos como smartwatches e smartphones com apps fitness, fazem, cada vez mais, parte dos acessórios utilizados pelo público das academias de ginástica, ou seja, o que antes eram recursos direcionados apenas à atletas, agora o apelo da indústria eletrônica é para o monitoramento de práticas relacionadas a um estilo de vida fisicamente ativo. Como exemplo podemos citar o aumento exponencial no número de downloads do aplicativo fitbit da rede de empresas da academia Bodytech; até o final de março de 2020, após liberação do acesso gratuito do seu app, foi identificado mais de $1.100 \%$ downloads [17].

Revisão sistemática e meta-analise realizada por Franssen et al. (2020) [18] avaliou a eficácia do uso de wearable technology no aumento do nível de atividade física e na modificação de indicadores de saúde metabólicos em população adulta com doenças crônicas. Em geral, indivíduos com wearable technology aumentaram seu nível de atividade física, e isso pode estar associado a melhorias na saúde cardiometabólica, incluindo circunferência da cintura, pressão arterial sistólica e concentração de lipoproteína de baixa densidade (LDL).

Para que a conexão da academia de ginástica com o cliente possa se diferenciar das propostas de plataformas digitais, além dos aspectos relacionados a interação social e supervisão profissional das atividades, um dos serviços que poderia ser melhor explorado seria um programa de avaliação física. Por meio de todo esse processo de elaboração e estruturação de programa de treinamento individualizado, com metas a serem cumpridas, utilizando como processo ações de intervenção na academia (off-line) e fora dela (online), passam a ser um diferencial.

Em levantamento realizado por Oliveira, Silva e Toscano (2014) [19] em 58 academias de Maceió-Al, foi observado, de maneira geral, que as avaliações até têm sido realizadas quando os clientes ingressam nas academias, no entanto, não há preocupação, na maioria das academias, em fazer um acompanhamento da evolução do cliente a partir dos dados avaliados inicialmente; destacando que apenas 19,0\% das academias observadas nesse estudo fizeram algum tipo de 
avaliação da aptidão cardiorrespiratória, considerado por vários especialistas como um dos indicadores da aptidão física mais importantes tanto para a saúde como para performance [20].

Outro destaque a ser considerado nessa conexão "off-line e online" das academias de ginástica com seus clientes se refere às recomendações sobre comportamentos sedentários, abordadas de forma mais intensa no âmbito de saúde pública, tendo sido apresentado propostas de intervenção com estratégias individuais e organizacionais [21].

Podemos exemplificar a importância do uso do controle individualizado de comportamentos sedentários primeiro com o estudo de Ekelund et al. (2016) [22], onde foi verificado que dependendo do tempo diário sentado, é preciso um volume diferenciado de exercícios para que esse possa ter impacto positivo na saúde. E mais, funções como o número de passos diários e avisos para interrupções no tempo sentado são funções presentes tanto em apps de fitness/saúde quanto nas wearable Technologies, podem funcionar como importantes estratégias para potencializar os resultados com a prescrição de exercícios individualizada, principalmente no que se refere a pessoas com doenças crônicas [23].

O presente estudo apresentou algumas limitações por ser um estudo transversal, com característica retrospectiva. Por se tratar de dados postados online, é possível que algum conteúdo tenha sido retirado antes da coleta, além da possibilidade de que comentários realizados naquele momento não terem sido explorados. No entanto, esse tipo de delineamento possibilita a formulação de hipóteses, a serem testadas em estudos posteriores. É importante destacar como delimitação do estudo que, apesar de 204 academias registradas no Conselho Regional de Educação Física - CREF19/AL no município de Maceió (dados reportados pelo CREF19/AL via e-mail em junho de 2020), fizeram parte do presente estudo oito academias que estavam distribuídas em suas regiões administrativas, sendo que em duas dessas regiões não foi possível incluir academias na amostra. Destacamos como positivo, a exploração de dados sobre uma temática que foi foco de bastante discussão durante o fechamento das academias na pandemia da COVID-19.

\section{CONCLUSÃO}

O cenário pós-pandemia para as academias de ginástica, assim como em vários outros segmentos da sociedade, deve passar por mudanças nos próximos anos. Apesar de previsões pessimistas no decorrer do isolamento social, quanto à adesão as práticas de atividade física, seja em ambientes aberto ou fechados, como em academias de ginástica, pesquisa de mercado aponta uma expectativa de aumento em 10 pontos percentuais em relação a voltar e/ou começar a frequentar academias de ginástica após a retomada com a normalidade.

Uma das tendências que deve se concretizar é a academia agregar serviços virtuais (online) ao seu portfólio físico (off-line). Em Maceió-AL, durante a pandemia, as academias investigadas começaram a fazer essa aproximação com seus clientes através dos meios eletrônicos, sendo as redes sociais o ambiente virtual predominantemente utilizado. Entretanto, é importante destacar que, apesar das plataformas de streaming especificas de programas de exercícios serem boas ferramentas ou recursos, não oferecem a atenção e sensação de pertencimento que as academias podem agregar ao seu serviço nessa conexão online / off-line.

Sendo assim, as academias em seus ajustes pós-pandemia, além dos cuidados óbvios na biossegurança, podem levar em consideração o grande interesse da população, durante a pandemia, com o exercício físico como fator de promoção à saúde e proteção à doença.

\section{REFERÊNCIAS BIBLIOGRÁFICAS}

1. OPAS. Brasil. Distanciamento social, vigilância e sistemas de saúde mais fortes são chaves para controlar pandemia de COVID-19, afirma diretora da OPAS. [Internet]. Disponível em: https://www.paho.org/bra/index.php?option=com_content\&view=article\&id=6188 [Acesso em 10 Jun 2020].

2. Diário Oficial da União. Portaria no 356, de 11 de março de 2020. Publicado em: 12 Mar 2020. Ed: 49; Seção:1, p.185. Ministério da Saúde do Brasil. Disponível em: https://www.conjur.com.br/dl/portariaministerio-saude-coronavirus.pdf [Acesso em 28 Mai 2020]. 
3. Diário Oficial. Estado de Alagoas. Decreto n 69.541, de 19 de março de 2020. (suplemento), Maceió, AL, ano 108, n.1287, p.1-4, 20 Mar 2020. Disponível em: http://www.imprensaoficialal.com.br/wpcontent/uploads/2020/03/DOEAL-20_03_2020-SUPLEMENTO.pdf [Acesso em 28 Mai 2020].

4. Chen P, Mao L, Nassis GP, Harmer P, Ainsworth BE, Li F. Coronavirus disease (COVID-19): The need to maintain regular physical activity while taking precautions. J Sport Health Sci. 2020;9(2):103-104, doi: 10.1016/j.jshs.2020.02.001.

5. ACSM. Staying physically active during the covid-19 pandemic. [Internet]. Disponível em: https://www.acsm.org/read-research/newsroom/news-releases/news-detail/2020/03/16/stayingphysically-active-during-covid-19-pandemic [Acesso em 23 May 2020].

6. CELAFISCS - - Centro de Estudos do Laboratório de Aptidão Física de São Caetano do Sul. Quarentena Sim! Sedentarismo Não! Informe n ${ }^{\circ}$ 1/Maio de 2020 [Internet]. São Paulo (SP): Programa Agita Ação; 15 Mai 2020 [acesso em 26 Jun 2020]. Disponível em: http://portalagita.org.br/pt/portalagita/noticias/item/quarentena-sim-sedentarismo-nao-2.html

7. Castro RRT, Silveira Neto JG, Castro RRT. Exercise training: a hero that can fight two pandemics at once. Int J Cardiovas Sci. 2020;33(3):284-87, doi: 10.36660/ijcs.20200083.

8. Guimarães SA. Quarentena: aplicativos para malhação em casa se multiplicam. [Internet]. Rio de Janeiro (RJ): Veja Rio; 1 Mai 2020 [atualizado em 22 Mai 2020; acesso em 19 Jun 2020]. Disponível em: https://vejario.abril.com.br/cidade/quarentena-aplicativo-malhacao-fit/

9. Toscano JJO. Academia de ginástica: um serviço de saúde latente. Rev Bras Ciên Mov. 2001;9(1):40-42.

10. IHRSA Global Report 2019. The state of the health club industry. [Internet]. Disponível em: https://www.ihrsa.org/publications/the-2019-ihrsa-global-report/\# [Acesso em 14 Jun 2020].

11. Williamson C, Baker G, Mutrie N, Niven A, Kelly P. Get the message? A scoping review of physical activity messaging. Int J Behav Nutr Phys Act. 2020;17(1):51, doi:10.1186/s12966-020-00954-3

12. Pitanga FJG, Beck CC, Pitanga CPS.Should Physical Activity Be Considered Essential During the COVID-19 Pandemic? Int J Cardiovasc Sci. 2020;33(4):401-403, doi: 10.36660/ijcs.20200072

13. Oliveira Neto L, Elsangedy HM, Tavares VDO, Teixeira CVLS, Behm DG, Da Silva-Grigoletto ME. \#TreineEmCasa - Treinamento físico em casa durante a pandemia do COVID-19 (SARS-CoV-2): abordagem fisiológica e comportamental. Rev Bras Fisiol Exerc 2020;19(2supl):S9-S19, doi: 10.33233/rbfe.v19i2.4006.

14. D'Angelo P. Prática de esportes na pandemia: o que mudou e como o brasileiro está se exercitando [Internet]. [place unknown]: Opinion Box; 20 de maio de 2020 [acesso em 23 Jun 2020]. Disponível em: https://blog.opinionbox.com/pratica-de-esportes-na-pandemia/

15. WHO. Global recommendations on physical activity for health. [Internet]. Geneva: World Health Organization; 2010 [acesso em 23 Jun 2020]. Disponível em: https://www.ncbi.nlm.nih.gov/books/NBK305057/

16. Thompson WR. Worldwide survey reveals fitness trends for 2020. ACSMs Health Fit J. 2019;23(6):1018, doi: 10.1249/FIT.0000000000000526

17. Suassuna F. Com isolamento, aplicativos de exercícios crescem $226 \%$ no Brasil [Internet]. [place unknown]: Metrópoles; 17 Abr 2020 [atualizado em 18 Abr 2020; acesso em 16 Jun 2020]. Disponível em: https://www.metropoles.com/vida-e-estilo/bem-estar/com-isolamento-aplicativos-de-exercicioscrescem-226-no-brasil

18. Franssen WMA, Franssen GHLM., Spaas, J. et al. Can consumer wearable activity tracker-based interventions improve physical activity and cardiometabolic health in patients with chronic diseases? A systematic review and meta-analysis of randomised controlled trials. Int J Behav Nutr Phys Act. 2020;17, 57, doi: 10.1186/s12966-020-00955-2.

19. Oliveira NM, Silva DAS, Toscano, JJO. Programas de avaliação em academias de ginástica: o que se faz? Rev Bras Ativ Fis e Saúde. 2014;19(5):566-68, doi: 10.12820/rbafs.v.19n5p568

20. Nieman DC. Exercício e Saúde: testes e prescrição de exercícios. 6ª ed. Barueri (SP): Manole, 2010. 816 p.

21. Manini TM, Carr LJ, King AC, Marshall S, Robinson TN, Rejeski WJ. Interventions to reduce sedentary behavior. Med Sci Sports Exerc. 2015;47(6):1306-1310, doi: 10.1249/MSS.0000000000000519

22. Ekelund U, Steene-Johannessen J, Brown WJ, et al. Does physical activity attenuate, or even eliminate, the detrimental association of sitting time with mortality? A harmonised meta-analysis of data from more than 1 million men and women. Lancet. 2016;388(10051):1302-10, doi: 10.1016/S0140-6736(16)303701

23. Hall KS, Hyde ET, Bassett DR, et al. Systematic review of the prospective association of daily step counts with risk of mortality, cardiovascular disease, and dysglycemia. Int $\mathrm{J}$ Behav Nutr Phys Act. 2020;17(1):78, doi: 10.1186/s12966-020-00978-9. 